\title{
Disease-modifying drugs for multiple sclerosis must be globally available according to therapeutic guidelines suitable to different regions of the world
}

\author{
Os medicamentos que modificam a história natural da esclerose múltipla devem ser \\ disponibilizados a todos os doentes segundo normas terapêuticas adequadas às \\ diferentes regiões do globo
}

Maria José Sá

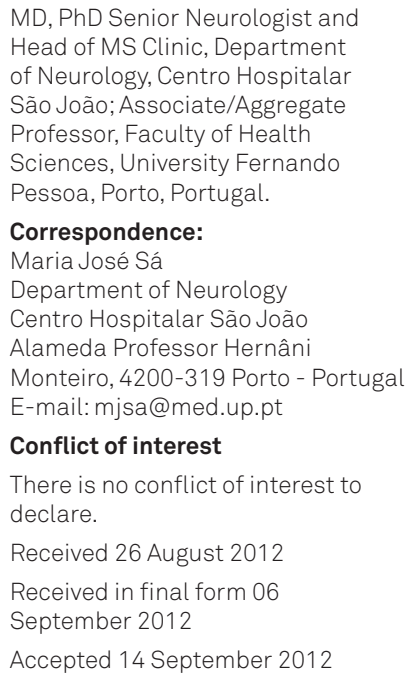

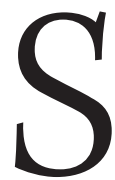
ince the early nineties of the XX century, multiple sclerosis (MS) moved drastically from a scarcely treated disease to a neurological chronic condition which is targeted by an increasing number of drugs that can significantly modify its natural evolution. These disease-modifying drugs (DMD) were specifically designed to intervene in the various inflammatory and immunological steps which underpin the MS pathogenesis, so their main indication is the relapsing-remitting (RR) form, and more recently, the clinically isolated syndromes (CIS). The first licensed drug was interferon beta 1-b, then formulations of interferon beta 1-a, glatiramer acetate, and, for aggressive forms, mitoxantrone. More recently natalizumab was introduced, and approximately one and a half year ago fingolimod was approved. Interferon beta and glatiramer acetate constitute the first-line group of DMD, being worldwide known due to the expected efficacy in clinical $(\approx 30 \%$ reduction in the annualized relapse rate) imaging (reduction in T2 lesion burden and in gadolinium-enhancing lesions) parameters, as well as in many other aspects: side effects; production of neutralizing antibodies to interferon, thus compromising its efficacy; need of adherence evaluation since these are self-injectable drugs which may lead to needle tiredness and perception of lower than expected efficacy after long-term therapy. Second-line DMD are significantly more efficient and its therapeutic administration regimens is easier, yet, its safety profile is worse, thus imposing strict vigilance programs: mitoxantrone is cardiotoxic and increases the risk of blood malignancies; natalizumab generates risk of progressive multifocal leukoencephalopathy, especially in patients previously submitted to immunosupressants with positive blood serology and after two-years of exposure; fingolimod, the first oral DMD approved for MS, needs strict cardiac monitoring and ophthalmologic assessment to exclude macular edema.

All DMD are expensive, representing a major cost to MS clinics and hospitals in countries in which the health system entirely reimburses them, the insurance companies and even the patients treated in private units in countries with strict health systems. The need for a health team specialized in MS, which would be able to provide frequent assistance, including patient and caregiver education, is determinant for a successful management, but it also would increase the economic burden of MS.

Before DMD, MS patients were treated with immunosupressants such as cyclophosphamide, azathioprine, methotrexate, and also with plasma exchange and human immunoglobulins, which non-specifically alter the immunological process, being still of off-label use in selected cases. Fortunately, MS patients now have a great therapeutic armamentarium that will surely be enriched in the near future, since numerous molecules being evaluated in many clinical trials. It is conceivable that the goal of an accurate personalized therapy according to the MS form and the patient idiosyncrasy in order to reach the disease-free 
activity status, defined by the absence of relapses and disability progression paralleled by the stable lesion burden, might be predicted in the future.

The increasing number of approved therapies, with distinct mechanisms of actions, indications, efficacy and safety profiles, lead the scientific community to establish therapeutic guidelines. It was recognized that DMD are not the same for all MS patients, neither in all stages, thus, similarly to other autoimmune diseases, such as rheumatoid arthritis, there is usually the need to escalate therapy. There are several clinical treatment guidelines for MS produced by consensus of expert groups in the USA $(2002)^{1}$, in the United Kingdom (NICE, $2003)^{2}$, and also internationally, $(2006)^{3}$, in Europe $(2008)^{4}$ and Spain $(2010)^{5}$. However, as elsewhere stated ${ }^{6}$, there is wide variation in the topics discussed and in the use of pharmacological agents; besides, therapeutic guidelines become quickly outdated in face of new drugs. For instance, fingolimod was considered only in recent consensus ${ }^{6,7}$.

In this line, the article of Finkelsztejn et al., published in this issue, presents the updated version of the 2010 Latin American $^{8}$ therapeutic algorithm for relapsing-remitting MS and for clinically isolated syndrome, and it adds very important information. Produced by the Latin American Multiple Sclerosis Forum, which includes experts from eight countries, the algorithm proposes four levels of therapy: first-line and second-line DMD, similar to others, third-line with alemtuzumab or rituximab for aggressive cases and failure of previous drugs, and fourth-line (autologous blood marrow transplant, cyclophosphamide) for especially severe situations; the role of mitoxantrone, other imunossupressants used off-label in MS and generic formulations is also debated; the features of each drug are presented in a practical way, highlighting indications, tolerability, side effects and safety aspects. The authors emphasize that the treatment for MS is a dynamic process due to the approval of new drugs, and their concepts regarding the place of different $\mathrm{DMD}$, therapeutic switch and escalation generally meet the guidelines designed by NorthAmerican and European experts. Yet, the Latin American Multiple Sclerosis Forum questions the direct applicability of those guidelines to Latin American countries, considering that the population of these countries have specific epidemiological, economic and sanitary characteristics. The article also addresses some important topics: pharmacovigilance systems and expenditure coverage; interest of supranational vigilance database for long-term appraisal; access of MS patients to DMD; pediatric MS; disease-free activity as a new therapeutic outcome; importance of cognitive and quality of life assessment with reliable scales.

\section{References}

1. Goodin DS, Frohman EM, Garmany GP Jr, et al. MD. Disease modifying therapies in multiple sclerosis: report of the Therapeutics and Technology Assessment Subcommittee of the American Academy of Neurology and the MS Council for Clinical Practice Guidelines. Neurology 2002;58:169-178.

2. National Collaborating Centre for Chronic Conditions. Clinical Guideline 8: Multiple sclerosis. Management of multiple sclerosis in primary and secondary care. National Institute for Clinical Excellence, London; 2003.

3. Karussis D, Biermann LD, Bohlega S, et al. A recommended treatment algorithm in relapsing multiple sclerosis: report of an international consensus meeting. Eur J Neurol 2006;13:61-71.

4. Multiple Sclerosis Therapy Consensus Group (MSTCG). Wiendl H, Toyka KV, et al. Basic and escalating immunomodulatory treatments in multiple sclerosis: current therapeutic recommendations. J Neurol 2008;255:1449-1463.
5. García Merino A, Fernández O, Montalbán X, de Andrés C, Arbizu T. Documento de consenso de la Sociedad Española de Neurología sobre el uso de medicamentos en esclerosis múltiple: escalado terapéutico. Neurología 2010;25:378-390.

6. Miller RM, Happe LE, Meyer KL, Spear RJ. Approaches to the management of agents used for the treatment of multiple sclerosis: consensus statements from a panel of U.S. managed care pharmacists and physicians. J Manag Care Pharm 2012;18:54-62.

7. Río J, Comabella M, Montalban X. Multiple sclerosis: current treatment algorithms. Curr Opin Neurol 2011;24:230-237.

8. Finkelsztejn A, Gabbai AA, Fragoso YD, et al. Latin American algorithm for treatment of relapsing-remitting multiple sclerosis using diseade-modifying agents. Arq Neuropsiquiatr 2012;70:799-806 